\title{
Paradigmas das práticas de gestão ambiental no segmento de produção de refeições no Brasil
}

\author{
Paradigms of environmental management \\ practices in the meal production sector in Brazil
}

Virgílio José Strasburg', Vanusca Dalosto Jahno²

口-

\section{RESUMO}

Os processos relacionados com a produção de refeições para os consumidores são causadores de impactos para o meio ambiente. Este trabalho teve como objetivo identificar quais são as práticas de restaurantes brasileiros relacionadas coma temática da gestão ambiental e da sustentabilidade. Trata-se deum estudo de revisão sistemática de publicações no período de 2000 a 2015. Entre os resultados encontrados, destacam-se a geração de resíduos de alimentos no processo de produção e consumo das refeições, além de investigações sobre acondicionamento, reciclagem e destinação desses resíduos. São apresentadas também as temáticas de utilização de recursos (água e energia) e um panorama sobre a avaliação dos aspectos ambientais por meio da prática de certificação de restaurantes comerciais com selos de sustentabilidade. Estudos avaliativos dessa natureza são importantes para contribuir para o diagnóstico da realidade no segmento de produção de refeições e também para fomentar investigações futuras que apontem para a redução dos impactos ambientais nessa atividade. Palavras-chave: impactos ambientais; gestão ambiental; refeições; restaurantes.

\begin{abstract}
The processes related to the production of meals for consumers cause impacts on the environment. The objective of this study is to identify the practices of Brazilian restaurants regarding to environmental management and sustainability. This is a systematic study reviewing publications from 2000 to 2015 . Among the findings, the generation of food waste in the process of production and consumption of food can be highlighted, as well as investigations on packaging, recycling and residues disposal. It also presents the themes of resources using (water and energy) and an overview of the evaluation of environmental aspects through the practice of certifying commercial restaurants with sustainability seals. Evaluative studies of this nature are important to contribute to the diagnosis of reality in meal production segment and also to promote further investigations that show the reduction of environmental impacts on this activity.
\end{abstract}

Keywords: environmental impacts; environmental management; meals; restaurants.

\section{INTRODUÇÃO}

A produção, a transformação, a distribuição e o consumo de alimentos são condições necessárias para a saúde e a prosperidade humana (VAN DER WERF et al., 2014). Durante a produção de refeições para coletividades ocorre uma série de processos que contemplam desde a seleção e o acondicionamento das matérias-primas até a preparação do produto acabado (ABREU; SPINELLI; ZANARDI, 2009). De acordo com a American Dietetic Association (ADA), essas etapas fazem parte de um conjunto de setores referentes à sustentabilidade nos sistemas alimentares (HARMON \& GERALD, 2007).
A produção de refeições em restaurante ocorre como um processo produtivo industrial, ou seja, com a entrada de insumos, o processamento destes e o produto final, que é a refeição, conforme esquematizado na Figura 1.

Nesse contexto, este estudo possui como problemática investigar quais são as práticas de restaurantes brasileiros em relação às abordagens com os aspectos de gestão ambiental e da sustentabilidade. Este estudo se justificou pela necessidade de compreender os aspectos e impactos da dimensão ambiental resultante das atividades desse importante segmento da economia presente diariamente na vida das pessoas.

1Doutor em Qualidade Ambiental pela Universidade Feevale. Professor Adjunto do Curso de Nutrição da Faculdade de Medicina da Universidade Federal do Rio Grande do Sul (UFRGS) Porto Alegre (RS), Brasil.

²Doutora em Ciências da Saúde pela Pontifícia Universidade Católica do Rio Grande do Sul (PUCRS). Professora Adjunta do Instituto de Ciências Exatas e Tecnológicas e Docente do Programa de Pós-Graduação em Qualidade Ambiental da Universidade Feevale - Novo Hamburgo (RS), Brasil.

Endereço para correspondência: Virgílio José Strasburg - Rua Ramiro Barcelos, 2.400, 40 andar - Santa Cecília - $90035-003$ - Porto Alegre (RS), Brasil - E-mail: vjs.nut@terra.com.br Recebido: 03/11/15 - Aceito: 27/05/16 - Reg. ABES: 155538 


\section{REFERENCIAL TEÓRICO}

\section{Mercado da alimentação}

A produção de refeição para coletividades ocorre em espaços específicos que podem receber nomes como Unidade de Alimentação e Nutrição (UAN), Unidade Produtora de Refeições (UPRs) ou Serviço de Alimentação Coletiva (SAC). Na contextualização do termo, são utilizadas, ainda, expressões como Unidades de Alimentação (UA), serviço de restauração e mercado de food service. O termo food service é aplicado para designar o fornecimento de refeições fora de casa (RODGERS, 2011).

O setor de alimentação coletiva é um mercado em plena ascensão. Nos Estados Unidos da América (EUA), dados da National Restaurants Association (NRA) projetaram um faturamento total de US\$ 783 bilhões, empregando 14,4 milhões de pessoas (NRA, 2016). No Brasil, dados agregados da Pesquisa de Orçamento Familiares (POF), realizada em 2008-2009, revelaram que as despesas com aquisição de alimentos fora do domicílio somaram $31,1 \%$ do orçamento familiar dos brasileiros, significando um crescimento de 7 pontos percentuais em relação ao período de 2002-2003 (IBGE, 2010). As pesquisas oficiais são as mais fidedignas em relação ao panorama das diversas regiões do país; no entanto, apresentam como limitação os períodos de levantamento e processamento de dados.

Segundo dados da Associação Brasileira das Empresas de Refeições Coletivas (ABERC), esse mercado como um todo, no ano de 2015, forneceu mais de 18,77 milhões de refeições/dia. Isso significa um movimento com cifras de R $\$ 31,58$ bilhões no ano. Para o ano de 2016, estima-se que essa quantidade deverá atingir cerca de 16,8 milhões de refeições diárias oferecidas por empresas com autogestão, refeições coletivas e refeições por convênio (ABERC, 2016).

\section{Gestão ambiental}

O termo gestão ambiental (GA), na definição de Rohrich e Cunha (2004), compreende um conjunto de políticas e estratégias administrativas e operacionais voltadas aos aspectos de prevenção do meio ambiente. Moretti, Sautter e Azevedo (2008) afirmam que a GA contempla uma série de procedimentos e medidas adequadamente definidos e aplicados com vistas a redução e controle dos impactos gerados por um empreendimento sobre o meio ambiente. Epelbaum (2004) destaca também a

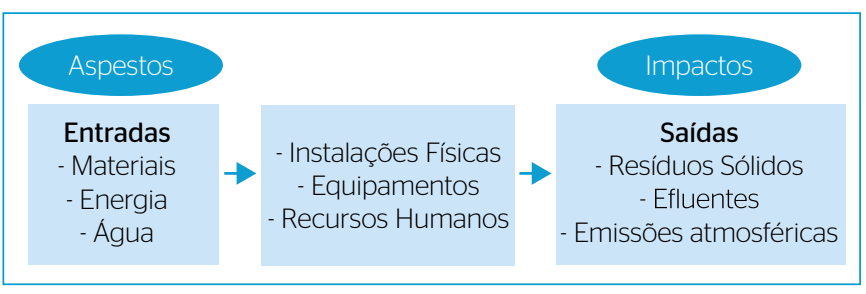

Figura 1 - Processo de produção de refeições.
GA como uma parte da gestão empresarial em que são necessárias as etapas de identificação, avaliação, controle e monitoramento, visando à redução dos impactos ambientais. Srivastra (2007) apresentou em seu estudo que a GA teve, inicialmente, por parte das empresas, uma visão voltada para a garantia da excelência ambiental direcionada para etapas como as de desenvolvimento de produtos, design dos processos e operações e gestão de resíduos, dentre outras.

Para Shigunov Neto et al. (2009), a GA está intimamente relacionada à gestão da qualidade mediante um conjunto de instrumentos $\mathrm{e}$ programas que visam proporcionar um processo de mudança organizacional e de melhoria contínua da qualidade ambiental de serviços, produtos e ambiente de trabalho em organizações públicas ou privadas, de qualquer porte. Dessa forma, fica “impossível desvincular a Gestão Ambiental da ciência administrativa, da gestão da qualidade e das organizações" (SHIGUNOV NETO et al., 2009, p. 03).

\section{Aspectos e impactos ambientais na produção de refeições}

Em qualquer atividade, para o desenvolvimento de um produto ou serviço, podem ser verificados os aspectos e os impactos ambientais. De acordo com a NBR ISO 14001, aspecto ambiental é definido como o “[...] elemento de atividades, produtos ou serviços de uma organização que pode interagir com o meio ambiente causando ou podendo causar impactos ambientais, positivos ou negativos [...]". Enquanto impacto ambiental é "[...] qualquer modificação do meio ambiente, adversa ou benéfica, que resulte, no todo ou em parte, dos aspectos ambientais da organização [...]" (ABNT, 2004a, p. 02).

As atividades das empresas que operam no segmento de produção de refeições giram em torno de dois componentes: a produção de comida e a prestação de serviços (LLACH et al., 2013). No fornecimento de refeições devem-se garantir as condições higiênico-sanitárias de alimentos preparados para o consumo humano. No Brasil essas recomendações estão citadas na Resolução RDC no 216, de 15 de setembro de 2004, que estabeleceu procedimentos de Boas Práticas para Serviços de Alimentação (ANVISA, 2004).

Dentre os elementos de verificação dessa resolução consta um subgrupo específico para o manejo de resíduos focado no correto acondicionamento; no entanto, não são contemplados os aspectos da redução ou minimização desses resíduos (ANVISA, 2004). Também na Política Nacional de Resíduos Sólidos (PNRS) constam, no artigo $7^{\circ}$, os aspectos de "não geração, redução, reutilização, reciclagem [...], bem como disposição final ambientalmente adequada dos rejeitos [...]" (BRASIL, 2010).

Na produção de refeições estão envolvidos muitos processos que causam impactos ambientais e também econômicos (HARMON \& GERALD, 2007). Nesse contexto, a ocorrência da geração de resíduos é inerente, e procedente das embalagens que fazem parte do acondicionamento 
de vários tipos de alimentos ou de produtos químicos que são utilizados direta e indiretamente na produção de refeições. Papéis, papelões, plásticos, vidros, latas e embalagens cartonadas (Tetrapack') são citados como materiais que, muitas vezes, não são separados adequadamente (WANG, 2012; GRAU, 2015).

Anteriormente ao fornecimento da refeição pronta, podem ocorrer desperdícios de matéria-prima alimentar. Dessa forma, a geração de resíduos pode ocorrer em várias fases, incluindo as etapas de recebimento, pré-preparo, preparo e distribuição. Resíduos na produção e no consumo de refeições são caracterizados, em especial, pela sua degradabilidade, sendo classificados pela norma NBR 10.004/2004 como sólidos domésticos, enquadrados na classe II não perigoso (ABNT, 2004b).

O desperdício de alimentos relacionado com a produção de refeições, segundo Vaz (2006), pode ser proveniente de sobras (alimentos produzidos e não distribuídos) e dos restos alimentares (quantidade de alimentos não consumidos e deixados no prato pelo cliente), sendo esses procedentes de etapas distintas desde o pré-preparo até o consumo final. Além da quantidade de alimentos desperdiçados, é necessário considerar a utilização de outros recursos intrínsecos, como a utilização de mão de obra, o uso de saneantes, água e energia.

Ao se considerar a finalidade de um restaurante, seja ele comercial ou institucional, com ou sem fins lucrativos, tem-se as atividades (aspectos) que causarão impactos ao ambiente (LLACH et al., 2013). É possível avaliar essa relação sob um olhar que contemple tanto os aspectos da gestão ambiental como também da qualidade sob uma abordagem integrativa. A adoção de práticas de gestão tanto de qualidade como ambientais pode ser crucial na competitividade, ou seja, o sucesso no mercado e o desempenho financeiro no setor de serviços (KARIM et al., 2007; SINGH; GARG; DESHMUKH, 2008; ALONSO-ALMEIDA, RODRÍGUEZ-ANTÓN; RUBIO-ANDRADA, 2012; LLACH et al., 2013).

Especificamente para a produção de refeições, Molina-Azorín et al. (2009) e Alonso-Almeida, Rodríguez-Antón e Rubio-Andrada (2012) argumentam que programas de gestão de qualidade nos quais sejam elencadas ações de padronização auxiliam na economia de tempo e dinheiro. Algumas práticas de qualidade e ambientais têm sido adotadas por inúmeras pequenas empresas como sendo uma estratégia de diferenciação em um mercado de grande concorrência (RUBIO-ANDRADA; ALONSO-ALMEIDA; RODRIGUEZ-ANTÓN, 2011; ALONSO-ALMEIDA \& RODRIGUEZ-ANTON, 2011).

Segundo Llach et al. (2013), os estudos realizados em práticas ambientais estão focados majoritariamente no setor industrial e nas grandes empresas. Esses autores realizaram um estudo em que aplicaram 374 inquéritos para gestores de empresas de pequeno porte no segmento de alimentação e bebidas, a fim de avaliar a adoção de práticas de qualidade e ambientais na região de Madri (Espanha) no ano de 2010. Os resultados do estudo mostraram que o segmento de restaurantes tem recebido pouca atenção em relação às práticas ambientais $\mathrm{e}$ que a associação entre a gestão ambiental e gestão da qualidade promove melhoria de competitividade e desempenho financeiro, além de melhorar a imagem dos estabelecimentos diante dos clientes (LLACH et al., 2013).

O crescimento da demanda de refeições fora do lar e da expansão do número de restaurantes impulsiona esse segmento para a adoção de práticas que preservem os recursos naturais e diminuam os impactos danosos ao ambiente (COSTELLO et al., 2009; FRIEL et al., 2009). Pelegrin (2011) destaca que as decisões empresariais tomadas pelos gestores de serviços de alimentação podem ter um impacto significativo sobre o meio ambiente e a economia. Ucker, Kemerich e Almeida (2012) corroboram afirmando ainda que a correta definição dos aspectos ambientais significativos é um dos passos mais importantes para o gerenciamento ambiental.

\section{METODOLOGIA}

Neste estudo, o método utilizado quanto à abordagem do problema é o qualitativo. Em relação aos procedimentos, é caracterizado como uma pesquisa bibliográfica secundária. Quanto à forma de explorar os dados, esta pesquisa será descritiva. Sendo um estudo qualitativo, a análise dos dados primou pelos conteúdos e pela categorização, buscando relacionamentos significativos entre as categorias que corroboraram os resultados apresentados ao final.

Na definição de Sampieri, Callado e Lucio (2013, p. 33), a abordagem qualitativa "[...] utiliza a coleta de dados sem medição numérica para descobrir ou aprimorar perguntas de pesquisa no processo de interpretação". Os mesmos autores destacam ainda que os dados qualitativos são "descrições detalhadas de situações, eventos pessoais, interações, condutas observadas e suas manifestações" (SAMPIERI; CALLADO; LUCIO, 2013, p. 34).

Dessa forma, a pesquisa bibliográfica secundária constitui-se de revisão em publicações científicas brasileiras e internacionais para o problema de pesquisa investigado. Foram utilizados os seguintes descritores para as buscas: gestão ambiental em restaurantes; sustentabilidade em restaurantes; e gestão de resíduos. Também foi tema de investigação a existência de selos de certificação de sustentabilidade ambiental em restaurantes. O recorte temporal compreende o período de 2000 a 2015.

\section{RESULTADOS E DISCUSSÃO}

\section{Geração de resíduos alimentares}

No segmento de fornecimento de refeições no Brasil, os principais achados de estudos estão relacionados com o desperdício de alimentos nos processos da produção ao consumo, conforme descrito no Quadro 1. 
As avaliações quantitativas dos resíduos gerados demandam um protocolo de mensuração e envolvimento das equipes de trabalho. Outro detalhe é que, quando esse tipo de trabalho é realizado para fins acadêmicos, as avaliações são pontuais de períodos específicos, como nos estudos que foram citados no Quadro 1.

O desperdício dos alimentos prontos para consumo pode ocorrer por falha de previsão no dimensionamento das quantidades preparadas ou ainda pelo hábito do usuário em servir mais do que necessita (VAZ, 2006). O desperdício de alimentos preparados pode estar associado ainda com questões comportamentais que envolvem os trabalhadores envolvidos nos processos, como também os usuários dos serviços. Goulart (2008) argumenta que não existem estudos conclusivos; por isso, estima-se que o desperdício com alimentos no setor de refeições coletivas chegue a $15 \%$.

Esse desperdício implica ainda na perda dos recursos que foram necessários para produzir os alimentos utilizados para compor a refeição, entre eles, água e energia (PIRANI \& ARAFAT, 2014). O monitoramento de resíduos é a ação que deve ser feita na busca pela redução desses danos. Dentre as possibilidades de monitoramento relacionadas com o controle de resto-ingestão, podem ser feitas medições quantitativas por tipos ou grupos de alimentos ou ainda a verificação visual (STRASBURG \& PASSOS, 2014; PIRANI \& ARAFAT, 2014).

Quanto ao processo de monitoramento de sobras de alimentos, estudo de Matias et al. (2013) em sete restaurantes da cidade de São Paulo identificou que o procedimento de controle de sobras nas cubas foi realizado por $57,1 \%$ dos estabelecimentos. Barthichoto et al. (2013) relataram, em sua pesquisa, que apenas 2 restaurantes, em um total de 32 avaliados, realizavam um procedimento para averiguar as sobras alimentares. Collares e Figueiredo (2012) diagnosticaram, no seu estudo, que os resíduos de alimentos provenientes de sobras, restos e rejeitos de pré-preparo corresponderam a $88 \%$ do total da composição gravimétrica em uma UAN. O trabalho de Van Waning (2010) mostrou que os restos de alimentos representaram $60,3 \%$ do total de resíduos gerados em serviços de hotelaria e restauração. Maragno, Trombin e Viana (2007) destacam que o lixiviado produzido pela decomposição de resíduos orgânicos é considerado a principal fonte de impactos ambientais danosos ao meio ambiente.

O segmento de cozinhas industriais e de restaurantes no Brasil trabalha ainda com muita matéria-prima in natura que necessita de beneficiamento (KAWASAKI; CYRILLO; MACHADO, 2007). Os procedimentos de seleção, pré-higienização e corte de alimentos acaba por gerar uma grande quantidade de resíduos orgânicos. $\mathrm{Na}$ Europa e nos EUA, as empresas desse segmento trabalham com os vegetais pré-processados; com isso, o trabalho na cozinha é o de finalização com processos térmicos (PROENÇA, 1999; RODGERS, 2007).
Quadro 1 - Estudos sobre desperdício de alimentos no fornecimento de refeições no Brasil.

\begin{tabular}{|c|c|c|}
\hline Autores & Características & Resultados \\
\hline $\begin{array}{l}\text { Augustini et al. } \\
\text { (2008) }\end{array}$ & $\begin{array}{c}\text { Restaurante de } \\
\text { empresa; período de } \\
20 \text { de abril a } 15 \text { de } \\
\text { maio de } 2007 \text { (14 dias); } \\
4.800 \text { refeições diárias } \\
\text { (almoço [A], jantar [J] e } \\
\text { ceia [C]). }\end{array}$ & $\begin{array}{c}\text { Sobras } \\
\text { A: de 7,48 a 13,39\%; J: de } \\
\text { 5,53 a 9.68\%; C: de 17,09 } \\
\text { a 60,85\%. } \\
\text { Resto-ingestão } \\
\text { A: 5,83\%; J: 6,87\%; C: 6,64\%. } \\
\text { Sobras e restos: } 9611,45 \\
\text { kg (total) (suficiente para } \\
\text { alimentar } 11.442 \text { pessoas } \\
\text { no período). }\end{array}$ \\
\hline $\begin{array}{l}\text { Ricarte et al. } \\
\text { (2008) }\end{array}$ & $\begin{array}{c}\text { Restaurante } \\
\text { universitário; período: } \\
\text { outubro e novembro } \\
\text { de } 2005 \text {. }\end{array}$ & $\begin{array}{c}\text { Sobras: } 203 \text { kg de frutas } \\
\text { e hortaliças (31,6\%); } \\
\text { Resto-ingestão: 8,39\% } \\
\text { (25 dias de avaliação) }\end{array}$ \\
\hline $\begin{array}{l}\text { De Moura et al. } \\
\text { (2009) }\end{array}$ & $\begin{array}{l}\text { Restaurante de } \\
\text { colégio agrícola; } \\
280 \text { refeições [A]. }\end{array}$ & $\begin{array}{l}\text { Resto-ingestão per } \\
\text { capita: } 58,44 \mathrm{~g} .\end{array}$ \\
\hline $\begin{array}{l}\text { Carneiro et al. } \\
\text { (2010) }\end{array}$ & $\begin{array}{l}\text { Restaurante universitário. } \\
\text { Caracterização dos } \\
\text { resíduos sólidos por } \\
\text { um período: setembro } \\
\text { e outubro de } 2009 \text { ( } 30 \\
\text { dias). Refeições: } 2.260 \\
\text { [A], [J] e [C] em dias úteis. }\end{array}$ & $\begin{array}{c}\text { Caracterização da } \\
\text { geração de resíduos: } \\
\text { 90\% do total de origem } \\
\text { orgânica (alimentares); } \\
\text { desses: } 64 \% \text { (6375,57 kg) } \\
\text { etapas de preparo e 36\% } \\
\text { (3599,33 kg) de restos de } \\
\text { cubas e bandejas. }\end{array}$ \\
\hline $\begin{array}{l}\text { Pedro \& Claro } \\
(2010)\end{array}$ & $\begin{array}{l}\text { Restaurante popular; } \\
\text { período: abril e maio de } \\
\text { 2009 (13 dias); } \\
\text { refeição [A]. }\end{array}$ & $\begin{array}{c}\text { Resto-ingestão (em } \\
\text { cinco dias): média de } \\
66,3 \text { kg desprezados } \\
\text { por dia. }\end{array}$ \\
\hline $\begin{array}{l}\text { Silva et al. } \\
(2010)\end{array}$ & $\begin{array}{l}\text { Hospital; período: } \\
\text { outubro e novembro de } \\
\text { 2008; refeições: } 100 \text { [A]. }\end{array}$ & $\begin{array}{c}\text { Resto-ingestão: de 39,4 a } \\
\text { 56,3 g/pessoa; } \\
\text { Após intervenção: } \\
28,4 \text { a 37,9 g. }\end{array}$ \\
\hline $\begin{array}{l}\text { Carmo \& Lima } \\
\text { (2011) }\end{array}$ & $\begin{array}{c}\text { Restaurante de } \\
\text { empresa; período: } \\
\text { fevereiro e março } 2011 \\
\text { (20 dias); } \\
140 \text { serviços [A]. }\end{array}$ & $\begin{array}{l}\text { Sobras de cubas: } 18 \\
\text { kg }(19,4 \%) \text { do total } \\
\text { produzido. }\end{array}$ \\
\hline $\begin{array}{l}\text { De Castro et al. } \\
\text { (2011) }\end{array}$ & $\begin{array}{l}\text { Colégio militar. Período: } \\
\text { setembro de } 2010 \text { (10 } \\
\text { dias). Refeições: } 900 \text { [A]. }\end{array}$ & $\begin{array}{c}\text { Sobras: } 212,36 \text { kg; 47,2\% } \\
\text { (média) } \\
\text { Resto-ingestão: 6,2\% }\end{array}$ \\
\hline $\begin{array}{l}\text { Leão et al. } \\
\text { (2011) }\end{array}$ & $\begin{array}{l}\text { Restaurante popular; } \\
\text { período de cinco dias. } \\
\text { Refeições: } 1.000 \text { [A]. }\end{array}$ & $\begin{array}{l}\text { Resto-ingestão per } \\
\text { capita: 7,8 g (média). }\end{array}$ \\
\hline $\begin{array}{l}\text { Machado et al. } \\
\text { (2012) }\end{array}$ & $\begin{array}{l}\text { Restaurante de empresa; } \\
\text { período: setembro e } \\
\text { outubro de } 2011 \text { (10 dias); } \\
1.350 \text { refeições diárias [A]. }\end{array}$ & $\begin{array}{l}\text { Resto-ingestão per } \\
\text { capita (média): 60,9 g } \\
\text { (antes de campanha); } \\
\text { 55,3 g (depois) }\end{array}$ \\
\hline $\begin{array}{l}\text { Carneiro } \\
\text { (2014) }\end{array}$ & $\begin{array}{l}\text { Restaurante de empresa; } \\
\text { período: maio de } 2011 \\
\text { (21 dias); } 2.000 \text { refeições } \\
\text { diárias: }[\mathrm{A}],[\mathrm{J}] \text { e }[\mathrm{C}] .\end{array}$ & $\begin{array}{c}\text { Resíduos de aparas } \\
\text { e preparo: 6375,57 kg } \\
\text { (56,2\%) } \\
\text { Restos e sobras: } 3599,33 \\
\text { kg (31,8\%) }\end{array}$ \\
\hline $\begin{array}{l}\text { Strasburg \& } \\
\text { Passos (2014) }\end{array}$ & $\begin{array}{c}\text { Restaurante de } \\
\text { empresa; período: } \\
\text { outubro de } 2010 \text { (14 } \\
\text { dias); } 520 \text { refeições } \\
\text { diárias [A] e [J]. }\end{array}$ & $\begin{array}{c}\text { Resto-ingestão per } \\
\text { capita: de 18,6 a 33,3 g. }\end{array}$ \\
\hline
\end{tabular}




\section{Gestão de resíduos}

A geração de resíduos é condição inerente ao segmento de produção de refeições, seja pelo uso das embalagens dos produtos utilizados ou pelos rejeitos de diversas naturezas no processamento dos alimentos. No entanto, a caracterização e o correto acondicionamento e destinação de tais restos de acordo com a PNRS (BRASIL, 2010) ainda constituem um desafio no contexto do segmento de restauração no Brasil.

Em estudo desenvolvido por Collares e Figueiredo (2012) foi realizada a avaliação e caracterização dos diferentes tipos de resíduos que ocorrem desde a etapa de recebimento até o consumo das refeições em um restaurante institucional. As autoras identificaram na composição gravimétrica dos resíduos sólidos os seguintes materiais: alimentos, plástico, papel/papelão, lata, madeira, pano e borracha (COLLARES \& FIGUEIREDO, 2012). Pesquisa de Rossi, Bussolo e Proença (2010), em um restaurante do setor hoteleiro, registrou que os resíduos foram classificados em quatro modalidades: orgânico, rejeitos, vidro e recicláveis. A relação desses materiais corrobora as informações de Zein, Wazner e Meylan (2008), que também classificam os resíduos sólidos nessas quatro últimas modalidades. Estudo em Dourados (MS), no qual foram entrevistados 20 estabelecimentos do segmento de restaurantes, foi identificado que os estabelecimentos pesquisados informaram realizar ao menos a destinação para a coleta seletiva de um dos seguintes itens: vidro, papelão e garrafas PET. Os demais tipos de resíduos tinham como destinação o aterro sanitário (ACRE \& CASTILHO, 2013). No estudo de Carneiro (2014) foi identificado que a quantidade de resíduos recicláveis (papel/papelão, vidros e latas) correspondeu a apenas $3,1 \%$ do total geral dos resíduos gerados em um restaurante institucional. Pospischek, Spinelli e Matias (2014) relatam, em seu estudo com 16 restaurantes comerciais na cidade de São Paulo, que 87,5\% dos estabelecimentos realizavam a coleta seletiva de resíduo reciclável (destes, apenas $18,8 \%$ utilizam recipiente com cores distintas para os recicláveis). Spinelli e Cale (2009) observaram, em seu estudo, que do total de resíduos gerados na produção de refeições em uma UAN, 87,8\% eram enviados para aterro sanitário, e 12,2\%, descartados para a coleta seletiva e reciclagem. Quanto à destinação dos resíduos orgânicos, os trabalhos de Barthichoto et al. (2013) e Matias et al. (2013) descreveram que os locais pesquisados encaminhavam esses restos para aterro sanitário mediante coleta do serviço municipal. Pospischek, Spinelli e Matias (2014) descreveram que a coleta dos resíduos nos 16 restaurantes comerciais pesquisados era realizada pela prefeitura $(43,8 \%)$ ou por cooperativas $(56,2 \%)$.

Rossi, Bussolo e Proença (2010) identificaram, nas inspeções em seu estudo, a inadequação em relação à correta separação dos resíduos, porém com um procedimento adequado para a coleta e destinação do óleo de cozinha usado. Outras experiências relataram o recolhimento de óleo de frituras por empresas ou pessoas cadastradas que desenvolviam subprodutos, como biodiesel e sabão (ACRE \& CASTILHO, 2013; BARTHICHOTO et al., 2013; MATIAS et al., 2013).

Harmon e Gerald (2007) descrevem que a ADA estabeleceu requisitos que orientam os nutricionistas sobre a responsabilidade profissional em relação aos aspectos de gestão de resíduos. Dentre esses requisitos estão o de minimizar o desperdício de alimentos, o de reciclar o óleo de cozinha utilizado em frituras e o de promover a coleta adequada e a reciclagem de materiais como vidro, metal, plástico, papelão, etc.

\section{Uso de recursos: água e energia}

Para que o fornecimento de refeições aconteça, o uso de recursos como a água e a energia elétrica é indispensável. A água entra em todas as etapas relacionadas com processos de limpeza e higienização, tanto de alimentos quanto de estrutura física, equipamentos e utensílios, além de ser constituinte para a cocção de várias preparações. O uso da energia elétrica é necessário para a conservação dos alimentos armazenados em cadeia fria (refrigeração e congelamento), como também para o funcionamento dos equipamentos utilizados para o preparo dos alimentos e a sua manutenção na distribuição em conservação a quente ou a frio.

A entidade Fecomércio (2010) estima que sejam utilizados de 20 a $30 \mathrm{~L}$ de água para o preparo de uma refeição em restaurantes comerciais. No entanto, no estudo de Souza, Santos e Santos (2012), realizado em restaurantes comerciais na cidade de Cascavel (PR), foi encontrado o consumo de 11 L de água por refeição servida (verificação de consumo mensal com hidrômetro). Os resultados de estudo de Barthichoto et al. (2013) mostraram que o consumo de água por refeição nos restaurantes que fizeram registros de monitoramento apresentou valores que variaram de 24 a $602,7 \mathrm{~L}$.

Pelo fato de a utilização da água estar relacionada com os processos diretos de produção e fornecimento de refeições, é indicado o seu uso de forma racional (OLIVEIRA, 2007; ABREU; SPINELLI; ZANARDI, 2009). Isso porque a utilização de grandes quantidades de água nas diversas etapas do processo produtivo de refeições é considerada como procedimento não sustentável (VEIROS \& PROENÇA, 2010; SOARES et al., 2011), além de refletir a necessidade de realizar medidas de educação com os colaboradores para o uso coerente da água (BARTHICHOTO et al., 2013).

A água é um bem precioso e indispensável para a vida do planeta. Por conta disso, a Organização das Nações Unidas (ONU) instituiu, no ano de 1992, o dia 22 de março como "Dia Mundial da Água”. O item sete dessa declaração descreve sobre o não desperdiçar tal recurso (CETESB, 2015). Quanto ao uso da água, a ADA faz recomendações quanto a estratégias de economia e reaproveitamento de água de cozimento, utilização de produtos de limpeza biodegradáveis e minimização da quantidade de resíduos descartados com a água (HARMON \& GERALD, 2007). 
Em estudo com restaurantes comerciais na cidade de São Paulo, apenas $37,5 \%(n=12)$ dos 32 estabelecimentos realizavam procedimento de mensuração de consumo de energia elétrica. As autoras do estudo mostraram que o consumo de energia elétrica por refeição variou de 0,2 a 1,3 kW.h ${ }^{-1}$ (BARTHICHOTO et al., 2013). Ainda nesse estudo foi destacado que $62,5 \%$ dos locais entrevistados declararam desenvolver com os funcionários ações de educação contra o desperdício de água e de energia elétrica, porém de maneira informal.

Quanto ao consumo de energia elétrica, estudo de Horovitz, realizado em 2008 (apud CHOU et al., 2012), destacou a pesquisa realizada pelo Pacific Gas \& Electric's Food Service Technology Center (FSTC), em que foi constatado que no segmento de varejo os restaurantes são os maiores usuários de energia elétrica, chegando a usar cinco vezes mais esse recurso por $\mathrm{m}^{2}$ em relação a outros empreendimentos comerciais. Stys (2008) destacou, em seu estudo, que os restaurantes dos EUA consomem um grande volume de produtos descartáveis, água e energia, com o custo anual de eletricidade e de gás na média de US\$ 161 por assento (vaga).

Também em uma definição mais voltada para a questão da GA em restaurantes, Oliveira (2007) sugere indicadores de monitoramento considerando:

- consumo energético em $\mathrm{kW} \cdot \mathrm{h}^{-1}$ por $\mathrm{m}^{2}$;

- consumo hídrico (volume/refeição); e

- volume de resíduo gerado (peso/volume/refeição).

Em relação à questão do uso de energia elétrica, a $\mathrm{ADA}$ faz orientações quanto a escolha de equipamentos com melhor eficiência energética, desenvolvimento de estratégias para a economia de energia e realização de manutenção preventiva nos equipamentos da unidade (HARMON \& GERALD, 2007).

De acordo com Blanco, Rey-Maquieira e Lozano (2009), uma redução no consumo de recursos ou na geração de resíduos é o primeiro incentivo para implementar práticas ambientais no setor de serviços. As empresas costumam adotar esse tipo de prática ambiental, a fim de obter economias em seus custos de consumo, uma vez que não exigem investimentos significativos, mas podem proporcionar um benefício financeiro imediato (ZENG et al., 2010). Alonso-Almeida, Rodríguez-Antón e Rubio-Andrada (2012) argumentam que reduções de desperdício de água e consumo de energia são situações em que ocorre a aproximação dos campos da gestão da qualidade com a GA.

$\mathrm{Hu}$, Parsa e Self (2010) destacam que as indústrias de alimentos e bebidas estão contribuindo para as questões ambientais mediante a redução de consumo de água e energia e da diminuição da geração de resíduos. No campo dos serviços de alimentação isso fica evidenciado com o crescimento e desenvolvimento dos "restaurantes verdes".

\section{Selos de sustentabilidade em restaurantes}

Algumas empresas de consultoria têm oferecido no mercado serviços de certificação em que são oferecidos "selos de estabelecimento sustentável”. Kipping e Kirkpatrick (2011) argumentam que nesse segmento são criadas demandas por serviços com base em uma "gestão do modismo".

O Quadro 2 apresenta os itens que são avaliados por algumas das empresas de consultoria que prestam esse tipo de certificação aos restaurantes no Brasil. Dos itens apresentados no Quadro 2, os elementos água, alimentos, energia, gestão de resíduos e materiais de operação tiveram as maiores frequências na abordagem de GA. No entanto, itens relacionados ao pessoal (funcionários) e de responsabilidade social também têm seu destaque, em uma abrangência mais ampla em relação à sustentabilidade.

No Brasil, de acordo com Melo et al. (2012), tem se observado que há restaurantes que estão procurando se adequar às questões de sustentabilidade. Isso se verifica na busca por uma culinária não necessariamente orgânica, mas pelos aspectos culturais envolvidos na alimentação com um cardápio mais brasileiro. Os autores citam também uma consultoria ambiental que criou o selo "Restaurante Sustentável" para empreendimentos gastronômicos, destacando sete restaurantes certificados na cidade de São Paulo no ano de 2011 (MELO et al., 2012). Acre e Castilho (2013) relatam outro tipo de experiência, que denominaram como ação de "marketing verde", e citam o caso de pousada da cidade de Bonito (MS) que utilizou essa divulgação como oportunidade de negócio.

No estudo de Novaes Botta e Donadone (2014), sobre consultorias em sustentabilidade, os autores identificaram que as empresas desse

Quadro 2 - Selos de sustentabilidade para restaurantes.

\begin{tabular}{|c|c|c|c|c|}
\hline \multirow{2}{*}{ Item avaliado } & \multicolumn{4}{|c|}{ Selos de Certificação } \\
\hline & EcoGourmet & Ecotest & $\begin{array}{l}\text { Green } \\
\text { Kitchen }\end{array}$ & $\begin{array}{l}\text { Restaurante } \\
\text { Sustentável }\end{array}$ \\
\hline Água & $x$ & $x$ & $x$ & $x$ \\
\hline $\operatorname{Ar}$ & & & $x$ & \\
\hline Energia & $x$ & $x$ & & $x$ \\
\hline Alimentos & $x$ & $x$ & $x$ & $x$ \\
\hline $\begin{array}{l}\text { Emissão de } \\
\text { carbono }\end{array}$ & X & & & \\
\hline $\begin{array}{l}\text { Gestão de } \\
\text { resíduos }\end{array}$ & $X$ & $x$ & $x$ & $x$ \\
\hline $\begin{array}{l}\text { Material de } \\
\text { operação } \\
\text { (construção) }\end{array}$ & $x$ & $x$ & $x$ & $x$ \\
\hline Pessoal & & $x$ & $x$ & $x$ \\
\hline Fornecedores & & $x$ & & $x$ \\
\hline $\begin{array}{l}\text { Responsabilidade } \\
\text { Social }\end{array}$ & $x$ & $x$ & & \\
\hline $\begin{array}{l}\text { Redução da } \\
\text { produção }\end{array}$ & & $x$ & & \\
\hline
\end{tabular}


segmento que atuam no Brasil utilizam o termo "sustentabilidade", com o embasamento do conceito do triple bottom line, ou seja, sob a perspectiva que contempla os aspectos ambientais, sociais e econômicos.

A preocupação empresarial em ter a palavra "sustentabilidade" associada às práticas de produtos e serviços se propagou mais fortemente no Brasil nos anos $2000 \mathrm{em}$ diante. As consultorias ambientais passaram a oferecer serviços contextualizando o termo "sustentável" como práticas de gestão empresarial de sucesso em longo prazo (NOVAES BOTTA \& DONADONE, 2014).

Por serem iniciativas pontuais, não existe atualmente um critério preestabelecido que defina os aspectos de avaliação ou algum padrão de exigência nas certificações ambientais, como ocorre nas certificações do padrão ISO, por exemplo. Para Oliveria (2007), o fato de se atentar para as questões ecológicas na gestão cotidiana de uma cozinha minimiza o impacto sobre o meio ambiente, podendo, também, garantir mais conforto aos comensais e operadores e difundir a sensibilidade e responsabilidade para as temáticas ambientais e socioculturais. Os itens elencados pelas empresas de consultoria que oferecem os serviços de selos ambientais vão de encontro aos pontos avaliados na certificação de restaurantes sustentáveis realizados pela Green Restaurant Association (GRA). A GRA é uma organização norte-americana que trabalha em um enfoque de sustentabilidade ambiental. A certificação para os restaurantes sustentáveis realizada pela GRA contempla a avaliação de sete categorias com os seguintes requisitos:

1. Eficiência no uso da água (uso de redutores de fluxo e equipamentos de menor consumo);

2. Redução de resíduos (reciclagem e compostagem);

3. Mobiliário sustentável e materiais de construção;
4. Comida sustentável (utilização de alimentos orgânicos, com certificação de bem-estar animal e de alimentos produzidos localmente);

5. Energia (equipamentos com selo de eficiência "energy star" e uso de fontes de energia limpa);

6. Utilização de descartáveis;

7. Produtos químicos (registrados em órgãos competentes) e redução de poluição (uso de fontes de energia limpa) (GRAU, 2015).

\section{CONSIDERAÇÕES FINAIS}

Este estudo fez um panorama sobre a temática da GA para o segmento de produção de refeições. Dos trabalhos encontrados podem ser destacados aspectos diretamente relacionados com os procedimentos de gestão. O enfoque dos estudos brasileiros é diferente da realidade e do escopo das temáticas investigadas em países desenvolvidos. Isso ficou evidente no contexto de abordagens relacionadas com questões de desperdício de recursos, especialmente os alimentos, envolvendo desde as etapas de preparação até as de consumo.

De forma semelhante, a adequada gestão da caracterização, separação e destinação dos resíduos gerados por parte das empresas desse segmento é importante para minimizar os impactos ao ambiente, como preconizado pela PNRS. A adequada gestão desses aspectos por parte das empresas desse segmento é importante para minimizar esses impactos ao ambiente e também para contribuir para o preconizado pela PNRS. Diante das inúmeras possibilidades de investigação sob essa temática, sugere-se a realização de mais estudos dessa natureza avaliativa, a fim de verificar um contexto de melhora em resultados futuros, apontando para a gestão de processos mais eficazes e que minimizem os impactos ambientais.

\section{REFERÊNCIAS}

ABERC - ASSOCIAÇÃO BRASILEIRA DAS EMPRESAS DE REFEIÇÕES COLETIVAS. (2016) Mercado Real. Disponível em: <http://www.aberc.com.br/mercadoreal.asp?IDMenu=21>. Acesso em: 27 abr. 2016.ABNT - ASSOCIAÇÃO BRASILEIRA DE NORMAS TÉCNICAS. (2004a) NBR ISO 14001: Sistemas de Gestão Ambiental - requisitos com orientações para uso. Rio de Janeiro: ABNT.

ABNT - ASSOCIAÇÃO BRASILEIRA DE NORMAS TÉCNICAS. (2004b) NBR 10.004/2004: Resíduos Sólidos - classificação. Rio de Janeiro: ABNT. 71 p.

ABREU, E.S.; SPINELLI, M.G.N.; ZANARDI, A.M.P. (Eds.) (2009) Gestão de Unidades de Alimentação e Nutrição: um modo de fazer. São Paulo: Metha. 342p

ACRE, D.M. \& CASTILHO, F.R. (2013) Gestão ambiental aplicado ao setor gastronômico: proposta para Dourados - MS. Revista Rosa dos Ventos, v. 5, n. 2, p. 248-263.
ALONSO-ALMEIDA, M.M. \& RODRIGUEZ-ANTÓN, J.M. (2011) Organizational behavior and strategies in adoption of certified management systems. An analysis of the Spanish hotel industry Journal of Cleaner Production, v. 19, n. 13, p. 1455-1463.

ALONSO-ALMEIDA, M.M.; RODRIGUEZ-ANTÓN, J.M.; RUBIO-ANDRADA, L. (2012) Reasons for implementing certified quality systems and impact on performance: an analysis of the hotel industry. The Service Industries Journal, v. 32, n. 5, p. 919-936.

ANVISA - AGÊNCIA NACIONAL DE VIGILÂNCIA SANITÁRIA (2004) Resolução RDC 216, de 15 de setembro de 2004. Disponível em: <http://www.ebah.com.br/content/ABAAAf4YIAH/ resolucao-rdc-216-04-bpf-servicos-alimentacao>. Acesso em: 17 jan. 2015.

AUGUSTINI, V.C.M.; KISHIMOTO, P.; TESCARO, T.C.; ALMEIDA, F.Q.A (2008) Avaliação do Índice de Resto-Ingesta e sobras em Unidade de Alimentação e Nutrição (UAN) de uma empresa metalúrgica na cidade de Piracicaba/SP. Revista Simbio-Logias, v. 1, n. 1, p. 99-110. 
BARTHICHOTO, M.; MATIAS, A.C.G.; SPINELLI, M.G.N.; ABREU, E.S. (2013) Responsabilidade ambiental: perfil das práticas de sustentabilidade desenvolvidas em unidades produtoras de refeições do bairro de Higienópolis, município de São Paulo. Qualit@s Revista Eletrônica, v. 14, n. 1, p. 1-12.

BLANCO, E.; REY-MAQUIEIRA, J.; LOZANO, J. (2009) Economic incentives for tourism firms to undertake voluntary environmental management. Tourism Management, v. 30, p. 112-122.

BRASIL. (2010) Lei no 12.305, de 2 de agosto de 2010. Disponível em: <http://www.planalto.gov.br/ccivil_O3/_ato2007-2010/2010/lei/ 112305.htm>. Acesso em: 23 jan. 2015.

CARMO, S.O. \& LIMA, T.P. (2011) Avaliação do índice de sobras limpas em uma unidade de alimentação e nutrição (UAN) institucional na cidade de Campo Grande-MS. Ensaios e Ciência: Ciências Agrárias, Biológicas e da Saúde, v. 15, n. 6, p. 9-20.

CARNEIRO, C.M.L.; LIMA, A.M.; AZEVEDO, J.B.; CASTRO, M.G.; SILVA, K.M.B. (2010) Diagnóstico dos resíduos sólidos produzidos no restaurante universitário da UFRN. In: ENCONTRO NACIONAL DE ENGENHARIA DE PRODUÇÃO, 30., 2010, São Carlos. Anais... São Carlos.

CARNEIRO, C.L. (2014) Gerenciamento integrado de resíduos sólidos e sua aplicabilidade em produção de refeições: um diálogo interdisciplinar. Holos, v. 30, n. 1, p. 68-74.

CETESB - COMPANHIA AMBIENTAL DO ESTADO DE SÃO PAULO. (2015) Declaração universal dos direitos da água. Disponível em: <http://www.cetesb.sp.gov.br/agua guas-superficiais/40-Declaracao-Universal-dos-Direitos--da--Água>. Acesso em: 23 mar. 2015.

CHOU, C-J; CHEN, K-S; WANG, Y-Y. (2012) Green practices in the restaurant industry from an innovation adoption perspective: Evidence from Taiwan. International Journal of Hospitality Management, v. 31, p. 703-711.

COLLARES, L.G.T. \& FIGUEIREDO, V.O. (2O12) Gestão de resíduos sólidos gerados na produção de refeições. Nutrição em Pauta, v. 114, p. 19-24.

COSTELLO, A.; ABBAS, M.; ALLEN, A.; BALL, S.; BELL, S; BELLAMY, R.; FRIEL, S.; GROCE, N.; JOHNSON, A.; KETT, M.; LEE, M.; LEVY, C.; MASLIN, M.; MCCOY, D.; MCGUIRE, B.; MONTGOMERY, H.; NAPIER, D.; PAGEL, C.; PATEL, J.; OLIVEIRA, J.A.P; REDCLIFT, N.; REES, H.; ROGGER, D.; SCOTT, J.; STEPHENSON, J.; TWIGG, J.; WOLFF, J.; PATTERSON, C (2009) Managing the health effects of climate change: Lancet and University College London Institute for Global Health Commission. The Lancet, v. 373, n. 9676, p. 1693-1733.

DE CASTRO, D.S.; RIBEIRO, D.M.A.; DUARTE, F.M.; MENDONÇA, X.M.F.D. (2011) Avaliação do Índice de Resto Ingestão de um Serviço de Alimentação e Nutrição Militar da Cidade de Belém, PA. Nutrição em Pauta, v. 1, n. 5, p. 46-49.

DE MOURA, P.N.; HONAISER, A.; BOLOGNINI, M.C.M. (2009) Avaliação do índice de resto ingestão e sobras em Unidade de Alimentação e Nutrição do Colégio Agrícola de Guarapuava (PR). Revista Salus, v. 3, n. 1, p. 15-22.

ECOGOURMET. Programa Ecogourmet. Disponível em <http:// www.programaecogourmet.com.br/>. Acesso em: 16 jan. 2015.
ECOTEST. Selo Ecotest. Disponível em: <http://www.seloecotest. com.br/categorias.html>. Acesso em: 16 jan. 2015.

EPELBAUM, M. (2004) A influência da Gestão Ambiental na Competitividade e no Sucesso Empresarial. Dissertação (Mestrado em Engenharia de Produção) - Universidade de São Paulo, São Paulo.

FECOMERCIO - FEDERAÇÃO DO COMÉRCIO DO ESTADO DE SÃO PAULO. (2010) O uso racional da água no comércio. Julho. Disponível em: <http://site.sabesp.com.br/uploads/file/asabesp_ doctos/cartilha_fecomercio.pdf>. Acesso em: O2 fev. 2015.

FRIEL, S.; DANGOUR, A.D.; GARNETT, T.; LOCK, K.; CHALABI, Z.; ROBERTS, I.; BUTLER, A.; BUTLER, C.D.; WAAGE, J.; MCMICHAEL, A.J.; HAINES, A. (2009) Public health benefits of strategies to reduce greenhouse-gas emissions: food and agriculture. The Lancet, v. 374, n. 9706, p. 2016-2025.

GOULART, R.M.M. (2008) Desperdício de alimentos: um problema de saúde pública. Integração, v. 54, n. 3, p. 285-288.

GREEN KITCHEN. Green Kitchen certificação. Disponível em: <http://www.greenkitchen.com.br/>. Acesso em: O4 fev. 2015.

GRAU - GREEN RESTAURANTS ASSOCIATION UNIVERSITY. (2O15) Green Restaurant Certification 4.0 Standards. Disponivel em: <http://www. dinegreen.com/restaurants/standards.asp>. Acesso em: O2 maio 2015.

HARMON, A.H. \& GERALD, B.L. (2007) Position of the American Dietetic Association: Food and Nutrition Professionals Can Implement Practices to Conserve Natural Resources and Support Ecological Sustainability. Journal of the American Dietetic Association, v. 107, n. 6, p. 1033-1043.

HOROVITZ, B. (2008). Can restaurants go green, earn green? USA Today. Disponível em: <http://www.usatoday.com/money/ industries/environment/2008-05-15-green-restaurants-ecofriendly_n.htm>. Acesso em: 16 jul. 2015.

HU, H.; PARSA, H.G.; SELF, J. (2010) The dynamics of green restaurant patronage. Cornell Hospitality Quarterly, v. 51, n. 3, p. 344-362.

IBGE - INSTITUTO BRASILEIRO DE GEOGRAFIA E ESTATISTICA. (2010). Pesquisa de Orçamentos Familiares 2008-2009. Aquisição alimentar domiciliar per capita Brasil e Grandes Regiões. Rio de Janeiro: Diretoria de Pesquisas. Coordenação de Trabalho e Rendimento.

KARIM, A.; SMITH, A.J.R.; HALGAMUGE, S.K.; ISLAM, M.M. (2007) A comparative study of manufacturing practices and performance variables. International Journal of Production Economics, v. 112, n. 2. p. $841-859$.

KAWASAKI, V.M.; CYRILLO, D.C.; MACHADO, F.M.S. (2007) Custo-efetividade da produção de refeições coletivas sob o aspecto higiênico-sanitario em sistemas cook-chill e tradicional. Revista de Nutrição, v. 2, n. 20, p. 129-138.

KIPPING, M. \& KIRKPATRICK, I. (2011) O desenvolvimento da consultoria de gestão empresarial entre a estrutura e a agência. In: DONADONE, J.C. \& JARDIM, M.A.C. (Org.). As centralidades e fronteiras das empresas do século 21. Bauru: Edusc. p. 249-285. 
LEÃO, G.S; MORAES, S.S.; MENDONÇA, X.M.F.D. (2011) Avaliação do Indice de Resto Ingestão e Aceitabilidade dos Cardápios Servidos no Restaurante Popular Municipal de Belém-PA. Nutrição em Pauta, v. 1, n. 2, p. 50-53.

LLACH, J.; PERRAMON, J.; ALONSO-ALMEIDA, M.M.; BAGUR-FEMENIAS, L. (2013) Joint impact of quality and environmental practices on firm performance in small service businesses: an empirical study of restaurants. Journal of Cleaner Production, v. 44, p. 96-104.

MACHADO, C. C. B.; MENDES, C. K.; SOUZA, P. G.; MARTINS, K. S. R.; SILVA, K. C. C. (2O12) Avaliação do índice de resto ingesta em uma Unidade de Alimentação e Nutrição institucional de Anápolis-GO. Ensaios e Ciência: ciências biológicas, agrárias e da saúde, v. 16, n. 6. p. 151-162.

MARAGNO, E.S.; TROMBIN, D.F.; VIANA, E. (2007) O uso da serragem no processo de minicompostagem. Engenharia Sanitária e Ambiental, v. 12, p. 355-60.

MATIAS, A.C.G.; ABREU, E.S.; BARTHICHOTO, M.; SPINELLI, M.G.N. (2013) Avaliação de práticas sustentáveis na produção de refeições segundo o tipo de gestão. Nutrição em Pauta, v. 21, n. 122, p. 25-29.

MELO, F.V.S.; BARBOSA, M.L.A.; FARIAS, S.A.; KOVACS, M.H.; MELO, S.R.S. (2012) Menu do dia: sustentabilidade - os consumidores estão deglutindo essa ideia? Revista de Gestão Social e Ambiental - RGSA, v. 6, n. 3, p. 96-112.

MOLINA-AZORIN, J.F.; CLAVER-CORTES, E.; PEREIRA-MOLINER, J.; TARI, J.J. (2009) Environmental practices and firm performance: an empirical analysis in the Spanish hotel industry. Journal of Cleaner Production, v. 17, p. 516-524.

MORETTI, G.N.; SAUTTER, K.D.; AZEVEDO, J.A.M. (2008) ISO 14001: implementar ou não? Uma proposta para a tomada de decisão. Revista Engenharia Sanitária, v. 13, n. 4, p. 416-425.

NRA - NATIONAL RESTAURANT ASSOCIATION. (2016) Restaurant Industry Forecast. Disponivel em: <http://www. restaurant.org/News-Research/Research/Forecast-2016>. Acesso: 27 abr. 2016.

NOVAES BOTTA, E.N. \& DONADONE, J.C. (2014) Consultorias em Sustentabilidade: polarizações e representatividades de um mercado crescente. Gestão \& Produção, v. 21, n. 4, p. 719-731.

OLIVEIRA, D.R. (2007) A cozinha politicamente correta - cozinha verde. Nutrição Profissional, v. 6, p. 30-34.

PEDRO, M.M.R. \& CLARO, J.A.C.S. (2010) Gestão de Perdas em Unidade de Restaurante Popular: Um Estudo de Caso em São Vicente. Qualit@s Revista Eletrônica, v.19. n.1, p.110.

PEREGRIN, T. (2011) Sustainability in Foodservice Operations: An Update. Journal of the American Dietetic Association, v.111, n. 9, p. 1286-1294.

PIRANI, S.I. \& ARAFAT, H.A. (2014) Solid waste management in the hospitality industry: a review. Journal of Environmental Management, v. 146, p. 320-336.
POSPISCHEK, V.S.; SPINELLI, M.G.N.; MATIAS, A.C.G. (2014) Avaliação de ações de sustentabilidade ambiental em restaurantes comerciais localizados no município de São Paulo. Demetra, v. 9, n. 2. p. 595-611.

PROENÇA, R.P.C. (1999) Novas tecnologias para a produção de refeições coletivas: recomendações de introdução para a realidade brasileira. Revista de Nutrição, v. 12, n. 1, p. 43-53

RESTAURANTE SUSTENTAVELL. Disponível em: <http:/l planetasustentavel.abril.com.br/noticia/desenvolvimento/ selo-restaurante-sustentavel-bar-sustentabilidade-certificacao-637597. shtml>. Acesso em: 18 fev. 2015.

RICARTE, M.P.R.; FÉ, M.A.B.M.; SANTOS, I.H.V.S.; LOPES, A.K.M. (2008) Avaliação do desperdício de alimentos em uma Unidade de Alimentação e Nutrição Institucional em Fortaleza-CE. Saber Científico, v. 1, n. 1, p. 158-175.

RODGERS, S. (2007) Innovation in food service technology and its strategic role. International Journal of Hospitality Management, v. 26, p. 899-912.

RODGERS, S. (2011) Food service research: An integrated approach. International Journal of Hospitality Management, v. 30, n. 2, p. 477-483.

ROHRICH, S.S. \& CUNHA, J.C. (2004) A proposição de uma taxonomia para a análise da gestão ambiental no Brasil. Revista de Administração Contemporânea, v. 8, n. 4, p. 86-95.

ROSSI, C.E.; BUSSOLO, C.; PROENÇA, R.C.P. (2O10) ISO 14000 no processo produtivo de refeições: Implantação e avaliação de um sistema de gestão ambiental. Nutrição em Pauta, v. 101, p. 49-54.

RUBIO-ANDRADA, L.; $\quad$ ALONSO-ALMEIDA, M.M.; RODRIGUEZ-ANTÓN, J.M. (2011) Motivations and impacts in the firm and stakeholders of quality certification: evidence from small and medium-sized service enterprises. Journal of Total Quality Management \& Business Excellence, v. 22, n. 8, p. 833-852.

SAMPIERI, R.H.; CALLADO, C.F.; LUCIO, M.P. (2013) Metodologia da pesquisa. 5. ed. Porto Alegre: Penso. 624p.

SHIGUNOV NETO, A.; CAMPOS, L.M.S.; SHIGUNOV, T. (2009) Fundamentos da Gestão Ambiental. Rio de Janeiro: Editora Ciência Moderna.

SILVA, A.M.; SILVA, C.P.; PESSINA, E.L. (2010) Avaliação do Indice de Resto Ingesta após campanha de conscientização dos clientes contra o desperdício de alimentos em um serviço de alimentação hospitalar. Revista Simbio-Logias, v. 3, n. 4, p. 43-56.

SINGH, R.K.; GARG, K.S.; DESHMUKH, S.G. (2008) Strategy development by SMEsfor competitiveness: a review. Benchmarking: An International Journal, v. 15, n. 5, p. 525-547.

SOARES, I.C.C; SILVA, E.R; PRIORE, S.E.; RIBEIRO, R.C.L; PEREIRA, M.M.L.S:; PINHEIRO-SANT'ANA, H.M. (2011) Quantificação e análise do custo da sobra limpa em unidades de alimentação e nutrição de uma empresa de grande porte. Revista de Nutrição, v. 24, n. 4, p. 593-604. 
SOUZA, D.P.; SANTOS, R.K.; SANTOS, R.F. (2012) Estimativa do consumo de água em restaurantes na cidade de Cascavel - PR. Acta Iguazu, v. 1, n. 3, p. 50-63.

SPINELLI, M. G. N. \& CALE, L. R. (2009) Avaliação de resíduos sólidos em uma unidade de alimentação e nutrição. Revista Simbio-Logias, v. 2, n. 1, p. 21-30.

SRIVASTARA, S.K. (2007) Green supply-chain management: a state-of-the-art literature review. International Journal of Management Reviews, v. 9, n. 1, p. 53-80.

STYS, B. (2008) Green restaurants: commercial kitchens face unique challenges as well as opportunities for saving energy and materials. Environmental Design \& Construction, v. 11, n. 5, p. 64.

STRASBURG, V.J. \& PASSOS, D. (2014) Avaliação do resto per capita de carnes e fatores associados em uma Unidade de Alimentação e Nutrição (UAN). Nutrição em Pauta, v. 22, n. 126, p. 46-50.

UCKER, F.E.; KEMERICH, P.D.C.; ALMEIDA, R.A. (2012) Indicadores ambientais: importantes instrumentos de gestão. Engenharia Ambiental, v. 9, n. 1, p. 119-127.

VAN DER WERF, H.M.G.; GARNETT, T.; CORSON, M.S.; HAYASHI, K.; CEDERBERG, C (2O14) Towards eco-efficient agriculture and food systems: theory, praxis and future challenges. Journal of Cleaner Production, v. 73, p. 1-9.

VAN WANING, A. (2010) Waste Characterization Study. [WWW Document]. Chicago: Department of Environment.. Disponivel em: $\quad$ <http://www.cityofchicago.org/dam/city/depts/doe/general/ RecyclingAndWasteMgmt_PDFs/WasteAndDiversionStudy/ WasteCharacterizationReport.pdf>. Acesso em: 18 fev. 2015.

VAZ, C.S. (2006) Restaurantes: controlando custos e aumentando Iucros. Brasília: Produção Independente. 196p.

VEIROS, M.B. \& PROENÇA, R.P.C. (2010) Princípios de Sustentabilidade na Produção de Refeições. Nutrição em Pauta, v. 102, p. 45-49.

WANG, R. (2012) Investigations of Important and Effective Effects of Green Practices in Restaurants. Procedia - Social and Behavioral Sciences, v. 40, p. 94-98.

ZEIN, K: WAZNER, M.S:; MEYLAN, G. (2008) Best Environmental Practices for the Hotel Industry [WWW Document]. Sustainable Business Associates. Disponível em: <http://www.sba-int.ch/spec/sba/download/ BGH/SBABGEHOTELLERIEENG2008.pdf>. Acesso em: 16 fev. 2015.

ZENG, S.X.; MENG, X.H.; YIN, H.T.; TAM, C.M.; SUN, L. (2010) Impact of cleaner production on business performance. Journal of Cleaner Production, v. 18, p. 975-983. 